\title{
Compensation of the Kondo effect in quantum dots coupled to ferromagnetic leads within equation of motion approach
}

\author{
Mariusz Krawiec \\ Institute of Physics and Nanotechnology Center, M. Curie-Skłodowska University, Pl. \\ M. Curie-Skłodowskiej 1, 20-031 Lublin, Poland \\ E-mail: krawiec@kft.umcs.lublin.pl
}

\begin{abstract}
We propose a new approximation scheme within equation of motion approach (EOM) to spin polarized transport through a quantum dot coupled to ferromagnetic leads. It has some advantages over a widely used in the literature standard EOM technique, in particular when we are interested in spin polarized quantities. Namely, it gives the values of the dot spin polarization which are closer to the ones obtained within numerical renormalization group (NRG), than the standard EOM approach. While restoring the Kondo effect, the spin polarization vanishes and the transport becomes unpolarized, in agreement with NRG and a real time diagrammatic calculations. The standard EOM procedure gives nonzero values of the spin polarization, and the transport is still spin polarized. Both approximations give the same correct splitting of the Kondo peaks due to ferromagnetism in the electrodes.
\end{abstract}

PACS numbers: 75.20.Hr, 72.15.Qm, 72.25.-b 


\section{Introduction}

The growing experimental interest in magnetic materials has evolved into a new research field, spintronics, where the transport properties are governed by the electron spin rather than charge. Thus, it opened new possibilities for technological progress in nanoelectronics [1, 2] and quantum computing [2, 3]. On the other hand, due to continuing experimental progress in miniaturization of electronic devices it became possible to study the fundamental problems of quantum mechanics. One of such examples is the Kondo effect [4] in quantum dots (QD).

The Kondo effect can be observed when the dot has unpaired spin or, in other words, has odd number of electrons. Thus the unpaired spin on the dot forms a many body singlet state with conduction electron spins in the leads. This state manifests itself in a resonance at the Fermi energy in the dot density of states and zero-bias maximum in differential conductance. The Kondo effect was predicted a long ago [5, 6, 7], extensively studied theoretically [8, 9, 10, 11] and confirmed in a series of beautiful experiments [12, 13, 14, 15, 16] in QD coupled to normal (non-magnetic) leads.

If the normal leads are replaced by ferromagnetic ones, spin degrees of freedom start to play significant role modifying transport [17, 18, 19, 20, 21, 22, 23, 24, 25, 26, [27, 28, 29, 30, 31, 32, 33, 34, 35] and thermoelectric properties [36], eventually leading to new phenomena. One of such new effects, associated with the ferromagnetism in the leads, is a splitting of the Kondo resonance due to spin dependent quantum charge fluctuations [20, 26, 27, 28, 33, 34, 35, 36. This splitting depends on the magnitude of the leads magnetizations as well as on their alignment in both leads. In particular, when the leads magnetizations point in opposite directions (anti-parallel alignment), one observes no splitting of the resonance and full Kondo effect is present for all spin polarizations. However while measuring differential conductance the Kondo resonance gets suppressed with increasing of the leads polarization, finally leading to a complete disappearance of zero-bias anomaly for fully polarized electrodes $(p=1)$. In this case transport is completely blocked and no current flows for any voltage. On the other hand, in parallel alignment, the Kondo resonance is split and also gets suppressed with increasing the leads polarization. However, in this case transport is not completely blocked even for $\mathrm{p}=1$, as one spin channel is still conducting [37].

Another interesting phenomenon is a compensation of the Kondo effect by external magnetic field $(B)$. As it was mentioned before, at zero magnetic field in parallel configuration, one observes splitting of the Kondo resonance. Moreover, finite spin polarization is induced on the dot due to ferromagnetic electrodes. It turns out, that applying external magnetic field one can recover full Kondo effect, i.e. no splitting of the zero-energy resonance and vanishing of spin polarization on the dot. The transport becomes unpolarized. Thus, at certain magnetic field $B=B_{\text {comp }}$, which we call it compensating field, the strong coupling limit is recovered. However, the problem is with standard equation of motion approach (EOM) [20, 36], as it gives non-zero spin polarization and spin polarized conductance, even at $B=B_{\text {comp }}$, i.e. when there is no 
splitting of the Kondo resonance. This is in contradiction with other approaches, like numerical renormalization group (NRG) [27] and a real-time diagrammatic technique [34], which correctly give zero spin polarization and equal spin dependent contributions to the conductance at $B=B_{\text {comp }}$. It is the purpose of the present paper to show how one can cure a standard EOM approach from their disabilities of non-zero spin polarization and spin polarized conductance at $B=B_{\text {comp }}$.

The paper is organized as follows: in Sec. 2 the model and details of calculations are presented. Section 3 shows a comparison of density of states and the on-dot occupations, obtained in different approaches. Section 4 is devoted to compensation of the Kondo effect within the standard and the present EOM approaches, and finally conclusions are given in Sec. 5.

\section{Model and formulation}

Our model system, i.e. quantum dot coupled to external leads is represented by a single impurity Anderson model in the limit of strong on-dot Coulomb repulsion $(U \rightarrow \infty)$. The model Hamiltonian in the slave boson representation [38, 36, 39, 40] reads

$$
\begin{aligned}
& H=\sum_{\lambda \mathbf{k} \sigma} \epsilon_{\lambda \mathbf{k} \sigma} c_{\lambda \mathbf{k} \sigma}^{+} c_{\lambda \mathbf{k} \sigma}+\sum_{\sigma} \varepsilon_{\sigma} f_{\sigma}^{+} f_{\sigma}+ \\
& \sum_{\lambda \mathbf{k}}\left(V_{\lambda \mathbf{k} \sigma} c_{\lambda \mathbf{k} \sigma}^{+} b^{+} f_{\sigma}+H . c .\right)
\end{aligned}
$$

where $\lambda=L(R)$ denotes left (right) lead, $c_{\lambda \mathbf{k} \sigma}^{+}\left(c_{\lambda \mathbf{k} \sigma}\right)$ is the creation (annihilation) operator for a conduction electron with the wave vector $\mathbf{k}$, spin $\sigma$ in the lead $\lambda, f_{\sigma}^{+}\left(f_{\sigma}\right)$ is a fermion operator, creating (annihilating) spin $\sigma$ on the dot, while $b^{+}(b)$ is a boson operator responsible for creating (annihilating) an empty dot state. The product of the fermion and boson operators gives a real dot electron operator $\left(d_{\sigma}=b^{+} f_{\sigma}\right) . V_{\lambda \mathbf{k} \sigma}$ is the hybridization between localized electron on the dot with the energy $\varepsilon_{\sigma}$ and conduction electron of energy $\epsilon_{\lambda \mathbf{k}}$ in the lead $\lambda$. Ferromagnetism of the electrodes is modeled via spin dependent conduction energy bandwidths. The constraint of no double occupancy is exactly taken into account by the non-canonical commutation rules for fermion and boson operators [38].

The total current $I=\sum_{\sigma} I_{\sigma}$, flowing through a quantum dot is given in the standard form [4]

$$
I=\frac{e}{\hbar} \sum_{\sigma} \int d \omega \frac{\Gamma_{L \sigma}(\omega) \Gamma_{R \sigma}(\omega)}{\Gamma_{L \sigma}(\omega)+\Gamma_{R \sigma}(\omega)}\left[f_{L}(\omega)-f_{R}(\omega)\right] \rho_{\sigma}(\omega),
$$

where $\Gamma_{\lambda \sigma}(\omega)=2 \pi \sum_{\mathbf{k}}\left|V_{\lambda \mathbf{k} \sigma}\right|^{2} \delta\left(\omega-\epsilon_{\lambda \mathbf{k} \sigma}\right)$ is the coupling parameter, $\rho_{\sigma}(\omega)$ is the ondot spin dependent density of states, and $f_{\lambda}(\omega)=f\left(\omega-\mu_{\lambda}\right)$ is the Fermi distribution function in the lead $\lambda$ with the chemical potential $\mu_{\lambda}$ and temperature $T$.

In order to get the density of states $\rho_{\sigma}(\omega)$ one has to calculate on-dot retarded Green function $(\mathrm{GF}) G_{\sigma}^{r}(\omega)$. Within equation of motion approach the resulted GF is 
Compensation of the Kondo effect in quantum dots coupled to ferromagnetic leads ... 4

[42, 43, 44, 45, 8, 46]

$$
G_{\sigma}^{r}(\omega)=\frac{1-\left\langle n_{-\sigma}\right\rangle}{\omega-\varepsilon_{\sigma}-\Sigma_{0 \sigma}(\omega)-\Sigma_{I \sigma}(\omega)}
$$

with non-interacting $(U=0)$

$$
\Sigma_{0 \sigma}(\omega)=\sum_{\lambda \mathbf{k}} \frac{\left|V_{\lambda \mathbf{k}}\right|^{2}}{\omega-\epsilon_{\lambda \mathbf{k} \sigma}+i 0^{+}}
$$

and interacting self-energy

$$
\Sigma_{I \sigma}(\omega)=\sum_{\lambda \mathbf{k}} \frac{\left|V_{\lambda \mathbf{k}}\right|^{2} f_{\lambda}\left(\epsilon_{\lambda \mathbf{k}-\sigma}\right)}{\omega-\epsilon_{\lambda \mathbf{k}-\sigma}-\varepsilon_{-\sigma}+\varepsilon_{\sigma}+i 0^{+}},
$$

which is responsible for the generation of the Kondo effect. The non-interacting selfenergy $\Sigma_{0 \sigma}(\omega)$ is an exact solution of the problem with no Coulomb interactions present. The interacting self-energy $\Sigma_{I \sigma}(\omega)$ is obtained by neglecting terms in the equation of motion for $G_{\sigma}^{r}(\omega)$ which cannot be directly projected onto original dot GF at this stage [47.

In the standard EOM approach the ferromagnetism in the leads is modeled via spin dependent coupling parameters $\Gamma_{\lambda \sigma}(\omega)=2 \pi\left|V_{\lambda}^{2}\right| \rho_{\lambda \sigma}\left(E_{F}\right)$, where $E_{F}$ is the Fermi energy in the lead $\lambda$. In order to get the splitting of the Kondo resonance due to ferromagnetism in the leads, one replaces bare dot energy level $\varepsilon_{\sigma}$ in self-energy $\Sigma_{I \sigma}(\omega)$ (Eq. 5) by renormalized one $\tilde{\varepsilon}_{\sigma}$, self-consistently found from the relation [20]

$$
\tilde{\varepsilon}_{\sigma}=\varepsilon_{\sigma}+\operatorname{Re}\left[\Sigma_{0 \sigma}\left(\tilde{\varepsilon}_{\sigma}\right)+\Sigma_{I \sigma}\left(\tilde{\varepsilon}_{\sigma}\right)\right] .
$$

In this way obtained splitting of the zero energy resonance remains in good agreement with a pour man scaling approach [20]. However such a procedure has very important drawback, when the Kondo effect is compensated by external magnetic field $B$. While at $B=B_{\text {comp }}$ there is no splitting of the Kondo resonance, as it follows from other approaches [20, 27, 34], it gives non-zero value of on-dot spin polarization and not equal spin polarized contributions to the transport, which is in contradiction with NRG [27] and the real time diagrammatic calculations [34].

To see why the standard EOM approach gives non-zero spin polarization at $B=B_{\text {comp }}$ let us examine the structure of the dot GF (Eq. (3)). At zero magnetic field $\left(\varepsilon_{\uparrow}=\varepsilon_{\downarrow}=\varepsilon_{0}\right)$ the denominator of GF can be written in the form

$$
\omega-\varepsilon_{0}-(1+p) \Sigma_{0}(\omega)-(1+p) \Sigma_{I}(\omega+\Delta \tilde{\varepsilon})
$$

for spin up electrons, and

$$
\omega-\varepsilon_{0}-(1-p) \Sigma_{0}(\omega)-(1-p) \Sigma_{I}(\omega-\Delta \tilde{\varepsilon})
$$

for spin down electrons, respectively. Both $\Sigma_{0}(\omega)$ and $\Sigma_{I}(\omega)$ contain spin independent couplings to the leads, and the spin dependence is shifted to the polarization parameter $p$, and $\Delta \tilde{\varepsilon}=\tilde{\varepsilon}_{\uparrow}-\tilde{\varepsilon}_{\downarrow}$ is calculated form Eq. (6). At $B=B_{\text {comp }}$ those equations are

$$
\begin{aligned}
& \omega-\varepsilon_{\uparrow}-(1+p) \Sigma_{0}(\omega)-(1+p) \Sigma_{I}(\omega) \\
& \omega-\varepsilon_{\downarrow}-(1-p) \Sigma_{0}(\omega)-(1-p) \Sigma_{I}(\omega)
\end{aligned}
$$


where $\varepsilon_{\sigma}=\varepsilon_{0}+\sigma B_{\text {comp }}$, and $\sigma \pm 1$. As one can notice, the real parts of both equations give the same values, that means the DOS for both spin directions will have a charge fluctuation resonance centered around the same energy. So in fact it should give the same occupations $n_{\uparrow}=n_{\downarrow}$. However, this is not the case, as we have to consider also the imaginary part of self-energies $\Sigma_{0}(\omega)$ and $\Sigma_{I}(\omega)$, which both depend on the polarization $p$. This leads to the smaller spin up occupation $n_{\uparrow}$ due to larger broadening of the charge fluctuation resonance in the DOS by factor $1+p$. Similarly, $n_{\downarrow}$ is larger due to a factor $1-p$ in the imaginary part of self-energies. As a result, spin polarization is non-zero at $B=B_{\text {comp }}$.

To cure those inconsistencies of the standard EOM technique, we propose modifications of the approach. We start from two requirements: (i) at zero magnetic field it should give the same splitting of the Kondo resonance as the standard EOM approach, and (ii) at $B=B_{\text {comp }}$ it should lead to zero spin polarization and no splitting of the Kondo resonance. This could be easily obtained if we skipped $1 \pm p$ factors in the imaginary parts of self-energies but left them in real parts. However such a procedure seems to be difficult to substantiate. Here, we propose slightly different approach which, by the construction, fulfills the above requirements. Namely, we use the same values of $\Gamma_{\lambda \uparrow}=\Gamma_{\lambda \downarrow}=\Gamma_{\lambda 0}$ for both spin directions but different spin dependent bandwidths $D_{\lambda \sigma}$ in the electrodes, which leads to spin asymmetry in the electrodes. This is one of the ways of modeling a ferromagnetism in the electrodes, closely related to the Stoner model, but not unique [27]. The splitting of the Kondo resonance due to ferromagnetic leads $\left(\Delta \tilde{\varepsilon}=\tilde{\varepsilon}_{\uparrow}-\tilde{\varepsilon}_{\downarrow}\right)$ is obtained in the same way as in standard EOM approach (Eq. 6) with replaced $\Gamma_{\lambda \sigma}$ in $\Sigma_{0 \sigma}$ and $\Sigma_{I \sigma}$ by $\tilde{\Gamma}_{\lambda \sigma}=\left(1+\sigma p_{\lambda}\right) \Gamma_{\lambda 0}$, where $p_{\lambda}$ is the polarization in the lead $\lambda$, and $\sigma= \pm 1$. Note that we use the same bare $\Gamma_{\lambda 0}$ for both spin directions in expression for the GF (Eq. (3i)). The polarization $p$ in the electrodes is calculated from spin dependent electron concentrations in the leads, i.e. $p=N_{\uparrow}-N_{\downarrow}$. Those concentrations will be different due to unequal bandwidths for spin up and spin down electrons. In this way obtained splitting of the zero energy resonance is the same as that obtained in pour man scaling and standard EOM technique [20]. Thus the requirement (i) is fulfilled.

To fulfill the requirement (ii) let us write down the denominator of the dot GF (Eq. (3)), similarly as for standard EOM equations. At zero magnetic field it gives

$$
\omega-\varepsilon_{0}-\Sigma_{0}(\omega)-\Sigma_{I}(\omega+\Delta \tilde{\varepsilon})
$$

for spin up electrons, and

$$
\omega-\varepsilon_{0}-\Sigma_{0}(\omega)-\Sigma_{I}(\omega-\Delta \tilde{\varepsilon})
$$

for spin down electrons, respectively. At $B=B_{\text {comp }}$ the above equations read

$$
\begin{gathered}
\omega-\varepsilon_{\uparrow}-\Sigma_{0}(\omega)-\Sigma_{I}(\omega) \\
\omega-\varepsilon_{\downarrow}-\Sigma_{0}(\omega)-\Sigma_{I}(\omega)
\end{gathered}
$$

where again $\varepsilon_{\sigma}=\varepsilon_{0}+\sigma B_{\text {comp }}$. As we can see, there is no splitting of the Kondo resonance, however, it also leads to non-zero spin polarization due to different real parts 
of the above equations. In this case the charge fluctuation resonances in spin polarized DOS are centered at different energies. Perhaps the easiest way of achieving zero spin polarization at $B=B_{\text {comp }}$ is to replace the original dot energies $\varepsilon_{\sigma}=\varepsilon_{0}+\sigma B$ in the above equations by the same energies $\varepsilon_{\sigma}$ but expressed in terms of $\tilde{\varepsilon}_{\sigma}$ and $\operatorname{Re} \Sigma_{I}\left(\tilde{\varepsilon}_{\sigma}\right)$, calculated from Eq. (宸). Thus at $B=0$ we get splitting of the Kondo resonance and non-zero spin polarization, while at $B=B_{\text {comp }}$ the splitting vanishes as well as the spin polarization. However, such a procedure is equivalent to leaving the original dot energy level $\varepsilon_{0}$ unchanged, even in the presence of the external magnetic field. In other words, we have to assume that the $B$ field modifies $\Sigma_{I}(\omega)$ only - there is no Zeeman splitting of the dot energy level.

Here we propose another, a more natural way of getting the zero spin polarization at $B=B_{\text {comp }}$. Namely, we replace the dot energies $\varepsilon_{\sigma}$ by $\tilde{\varepsilon}_{\sigma}$, not only in the interacting self-energy $\Sigma_{I \sigma}$ (Eq. 5), as in standard EOM, but also in full retarded Green function $G_{\sigma}^{r}$ (Eq. 3). This corresponds to the same replacement of the energies in Eqs. (13) and (14), which in turn leads to the same expressions for Green functions for both spin directions at $B=B_{\text {comp }}$. Such a renormalization of $\varepsilon_{\sigma}$, in a heuristic way, represents fact that higher order GFs in EOM procedure give also $V^{2}$ contributions (similar to $\left.\Sigma_{I \sigma}(\omega)\right)$ going like $\ln (\omega)$ near the Fermi energy [46], which are neglected in standard $V^{2}$ EOM approach. Perhaps this is a simplest way of enhancing spin correlations, i.e. a transfer of the spectral weight from charge to spin sector by the renormalization of the dot energy level. This in turn leads to better description of the Kondo effect, as it will be shown later on (see discussion in Sec. 3). Such a procedure gives the correct splitting of the Kondo resonance $\Delta \tilde{\varepsilon}$, equal spin dependent contributions to the conductance as well as the vanishing of the spin polarization at $B=B_{\text {comp }}$. Thus both requirements (i) and (ii) are automatically fulfilled. Of course, such a modification does not cure all the shortcomings of standard EOM approach, like basic Fermi liquid relations, which are little bit less but still violated. However, it leads to better qualitative description of the Kondo effect in quantum dots.

In the following we show how those modifications of the approach influence the properties of the quantum dot in the presence of ferromagnetism. In numerical calculations all the energies are measured with respect to the Fermi energy $E_{F}=0$ in units of $\Gamma=\Gamma_{L 0}+\Gamma_{R 0}=1$.

\section{Density of states}

The most influenced quantity by the above modifications of the EOM approach is the density of states (DOS) $A_{\sigma}(\omega)=-\frac{1}{\pi} G_{\sigma}^{r}(\omega)$.

Figure 1 shows a comparison of spin dependent densities of states obtained in the standard EOM (top panel) and in the present, modified EOM approach (bottom panel). First of all, one can see that the positions of the Kondo resonances remain unchanged in both approaches, giving the same value of the splitting. However, there is a change in their spectral weights. While in the standard EOM the Kondo resonance for 
Compensation of the Kondo effect in quantum dots coupled to ferromagnetic leads ... 7
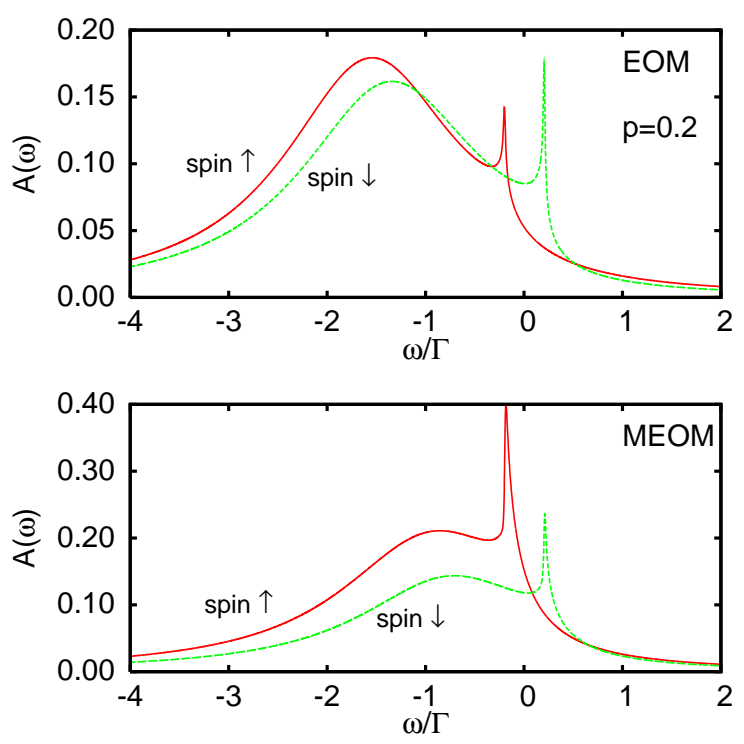

Figure 1. Comparison of spin up (solid line) and spin down (dashed line) dot density of states obtained in the standard EOM (top panel) and in the present approach (bottom panel). The model parameters are: $\mathrm{T}=0.005, \varepsilon_{\sigma}=-2$ in units of $\Gamma$. The polarization of the electrodes is $\mathrm{p}_{L}=\mathrm{p}_{R}=0.2$.

spin down electrons is higher, in the modified equation of motion technique (MEOM) the resonance for spin up electrons is more pronounced, similarly as in real time diagrammatic calculations [34]. Moreover, the DOS around the dot energy level $\varepsilon_{\sigma}$ also changes leading to higher values for spin up electrons. This is also in agreement with real time diagrammatic approach (see Fig. 6 of Ref. [34]). At the same time the standard EOM gives comparable or only slightly higher DOS around $\varepsilon_{\sigma}$. At the first sight, the higher Kondo resonance for spin up electrons seems to be counterintuitive, as there is more spin up electrons in the leads, thus they should lead to better screening of spin down electrons on the dot. However, one has to remember that due to spin dependent renormalization of the dot energy level, the spin up electron occupation on the dot is larger, thus the cotunneling processes, including Kondo ones, are more efficient in spin up channel.

As one can notice, the MEOM approach seems to give to large renormalization of the dot energy level $\varepsilon_{\sigma}$, shifting the charge fluctuation resonance in the DOS towards the Fermi energy for both spin directions (compare both panels of Fig. 1). However such a renormalization influences also the DOS around $E_{F}$ leading to better description of the Kondo resonance. This can be seen in Fig. 2, where the densities of states for unpolarized leads $(\mathrm{p}=0)$ obtained in different approaches are shown. The solid line is obtained within non-crossing approximation (NCA) [48], which is a widely accepted and reliable technique for description of the Kondo problem in the case of non-magnetic leads and in the lack of external magnetic field [10, 49, 50, 11], the dashed one within standard EOM, while the dotted one within the present approach. Clearly, the present approach 
Compensation of the Kondo effect in quantum dots coupled to ferromagnetic leads ... 8

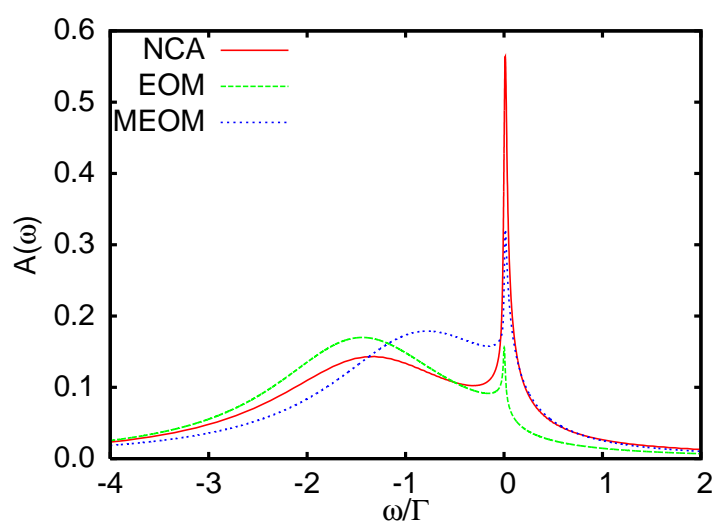

Figure 2. The dot density of states obtained in NCA (solid line), standard EOM (dashed line) and modified EOM (dotted line) approach. The model parameters are the same as in Fig. 1 but the electrodes are unpolarized now $(\mathrm{p}=0)$.

gives better behavior of the DOS in the low energy regime, thus better description of the Kondo effect.

Physically, as it was previously mentioned, the renormalization of $\varepsilon_{\sigma}$ in GF represents $V^{2}$ contributions to GF, which are obtained while calculating higher order GFs in EOM procedure. In particular, performing an effective $V^{4}$ order EOM calculations, one gets $V^{2}$ contributions, going like $\ln (\omega)$ around the Fermi energy [46]. Those contributions are very important for the Kondo effect, as they represent inelastic scattering processes leading to the broadening of the Kondo resonance (compare Fig. 2 and Fig. 1 of Ref. [46]). Here similar effect is achieved simply just by renormalization of the dot energy level, which in turn leads to a transfer of the spectral weight form charge to spin sector enhancing the Kondo effect. Furthermore, this can be qualitatively explained even within the EOM approach. Namely, while calculating original QD Green function one obtains, usually neglected, an energy independent term $\Lambda=-\sum_{\lambda \mathbf{k}} V_{\lambda \mathbf{k}}\left\langle f_{-\sigma}^{+} b c_{\lambda \mathbf{k}-\sigma}\right\rangle$, which shifts the QD bare energy level towards $E_{F}$. In equilibrium, the $\Lambda$ can be expressed in terms of the dot GF, i.e. $\Lambda=\frac{1}{\pi} \sum_{\lambda \mathbf{k}} V_{\lambda \mathbf{k}}^{2} \int d \omega f(\omega) \operatorname{Im}\left(\frac{G_{-\sigma}^{r}(\omega)}{\omega-\epsilon_{\lambda \mathbf{k}-\sigma}}\right)$. Clearly, this is $V^{2}$ contribution renormalizing QD energy level in the same way as we propose here. This contribution has to be also calculated self-consistently, as it depends on the QD energy level, and explains why we replaced original QD energy level $\varepsilon_{\sigma}$ by $\tilde{\varepsilon}_{\sigma}$ also in full retarded QD Green function $G_{\sigma}^{r}(\omega)($ Eq. (3) $)$.

Another important consequence associated with the above modifications of the EOM approach is the average dot occupation $n=n_{\uparrow}+n_{\downarrow}$. For unpolarized leads and the dot energy level $\varepsilon_{\sigma}=-2$, the MEOM gives $n_{M E O M}=0.87$ which is close to value obtained within NCA $\left(n_{N C A}=0.85\right)$, while the standard EOM deviates from $n_{N C A}$ by almost $10 \%$, giving $n_{E O M}=0.93$. 


\section{Recovery of the Kondo effect}

Now, let us discuss the effect of the external magnetic field on the Kondo effect. In the following we assume that the external magnetic field acts on the dot spin only and disregard its influence on the properties of the leads. In real experiment, this cannot be neglected, as it can lead to the modifications of the magnetic properties and the density of states in the electrodes [51]. However, it needs fully self-consistent calculations, which are out of the scope of the present work.

It turns out that applying magnetic field one can recover the Kondo effect. At certain field $B=B_{\text {comp }}$ there is no splitting of the zero energy resonances (in real experiments the $B$ field will also modify DOS in the electrodes [51]), the transport is unpolarized, and the spin polarization should vanish in this case, so the strong coupling limit is reached. Thus, full Kondo effect is recovered. Those conclusions have been obtained within NRG [27] and a real time diagrammatic technique [34]. Also standard EOM approach gives no splitting of the Kondo resonance in this case [20] but does not fulfill the conditions of vanishing spin polarization and equal spin dependent contributions to the conductance at $B_{\text {comp }}$. The proposed modifications of the standard EOM substantially improve the results leading also to unpolarized transport and to zero spin polarization at $B_{\text {comp }}$.

Figure 3 shows a comparison of the spin polarization $n_{\uparrow}-n_{\downarrow}$ vs. magnetic field $B$, calculated within the present approach (solid line) and the standard EOM technique (dashed line). The dotted line represents the splitting of the zero energy resonance $\Delta \tilde{\varepsilon}_{\sigma}$.

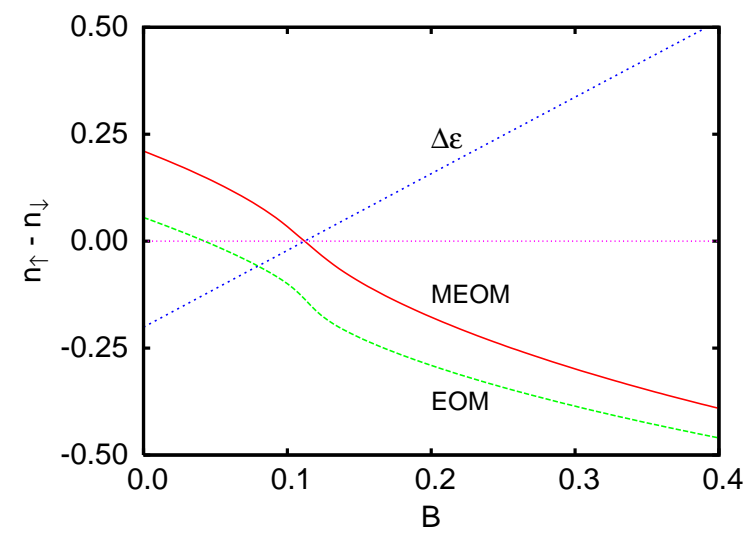

Figure 3. The spin polarization $n_{\uparrow}-n_{\downarrow}$ calculated within the present approach (solid line) and within standard EOM (dashed line). Note that within MEOM approach spin polarization vanishes at $B=B_{\text {comp }}=0.11$, while EOM gives non-zero value of it. The splitting of the zero energy resonance is also shown (dotted line). The model parameters are the same as in Fig. 1.

It is clearly seen that the spin polarization obtained within MEOM vanishes, while standard EOM gives non-zero value of it at $B=B_{\text {comp }}=0.11$, for which $\Delta \tilde{\varepsilon}_{\sigma}=0$. At this field standard EOM gives $n_{\uparrow}-n_{\downarrow}=-0.135$. The spin polarization vanishes at 
much smaller $B$ field, i.e. at $B=0.042$.

The non-zero spin polarization results form different densities of states for spin up and spin down electrons, which can be seen in Fig. 4 (dashed and dotted line) or Fig. 1(d) of Ref. [20]. Clearly, the spin down DOS is much larger than spin up one, even if

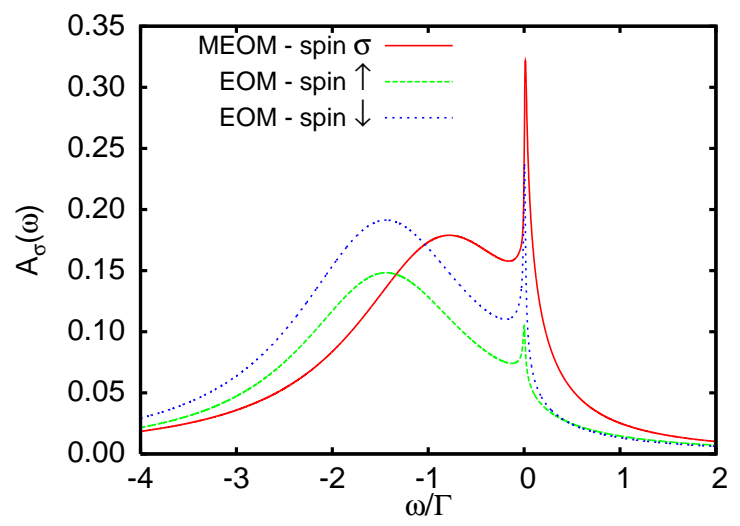

Figure 4. Spin dependent density of states at $B=B_{\text {comp }}$. Modified EOM gives exactly the same DOS for both spin directions (solid line), while the standard EOM gives different densities of states for spin up (dashed line) and spin down electrons (dotted line). The parameters are the same as previously used.

there is no splitting of the Kondo resonance. Similarly, other approaches, like NRG or real time diagrammatic approach, give also asymmetric density of states but at the same time they give zero spin polarization. This is achieved in the following way. Spin down electrons have larger DOS at the Fermi energy but smaller around $\varepsilon_{\sigma}$, in comparison with spin up electrons, so the resulting DOS integrated with the Fermi distribution functions give the same occupations, thus no spin polarization [27, 34]. The MEOM approach also gives zero spin polarization but the price we have to pay for this are the same densities of states for both spin directions (see solid line in Fig. 4).

It is interesting to see the behavior of the dot spin-dependent occupations as a function of the leads polarization in both approaches. Corresponding spin up, spin down and the total occupations are shown in Fig. 5 at zero magnetic filed (left panels) and at $B=0.05$ (right panels). The top panels represent occupations obtained with help of standard EOM, while bottom panels - those obtained by the present, modified EOM approach. As one can see, at $B=0$ both approaches give $n_{\uparrow}=n_{\downarrow}$ for unpolarized leads $(\mathrm{p}=0)$. However, the difference between $n_{\uparrow}$ and $n_{\downarrow}$, (spin polarization) obtained in the standard EOM is much smaller than in the modified EOM approach. Unfortunately, both approaches give too small values of spin polarizations in comparison to the other approaches [27, 34]. At finite magnetic field, modified EOM gives $n_{\uparrow}=n_{\downarrow}$ for a finite $\mathrm{p}$, for which $\Delta \tilde{\varepsilon}_{\sigma}=0$. On the other hand, the standard EOM gives $n_{\uparrow} \neq n_{\downarrow}$, in contradiction to NRG results (compare Fig. 1 of Ref. [27]).

While the standard EOM approach gives worse (smaller) values of spin polarization, it gives better behavior of the total occupation $\left(n_{\uparrow}+n_{\downarrow}\right)$ vs. leads polarization. In the 

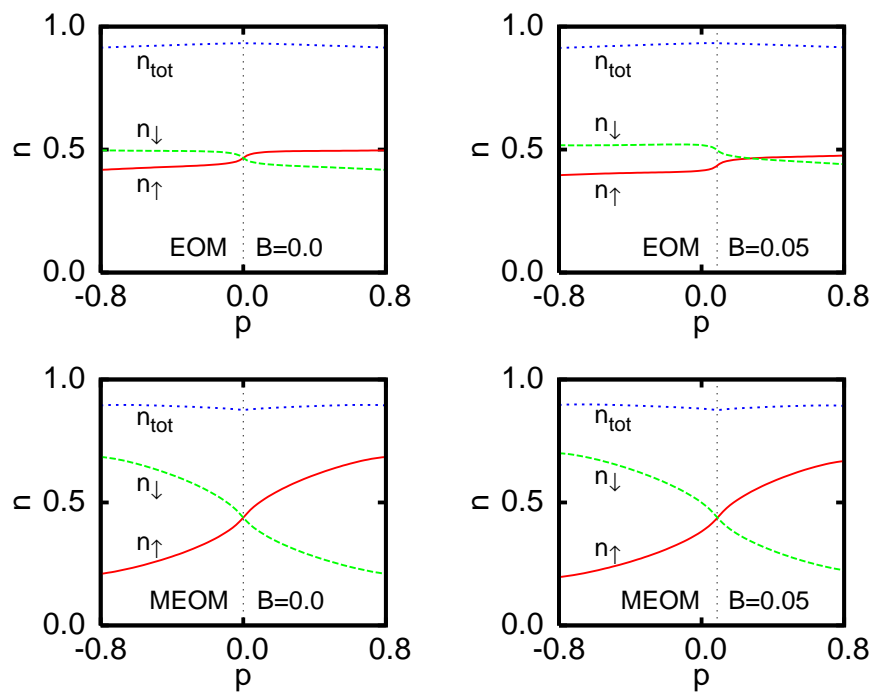

Figure 5. Spin-dependent occupation of the $\operatorname{dot}$ at $B=0$ (left panels) and $B=0.1$ (right panels) as a function of spin polarization p. Top panels represent results obtained within standard EOM, while bottom ones - within the present approach. At $B=0$ both approaches give $n_{\uparrow}=n_{\downarrow}$ for $\mathrm{p}=0$. At $B=0.05 \mathrm{MEOM}$ gives $n_{\uparrow}=n_{\downarrow}$ for a finite $\mathrm{p}$, for which $\Delta \tilde{\varepsilon}_{\sigma}=0$, while the EOM gives $n_{\uparrow} \neq n_{\downarrow}$ in this case.

standard EOM approach the total occupation weakly increases with decreasing of the leads polarization $\mathrm{p}$ (top panels of Fig. 5), surprisingly giving a correct position of the maximum of the total occupation, in agreement with NRG results [27]. In the present approach, the situation is opposite, namely, the total occupation weakly decreases with decreasing of the leads polarization, giving a correct position of a minimum, not a maximum of the total occupation. In both cases, however, the changes of the total occupations due to the leads polarizations are very small.

Finally, let us turn our attention to the transport properties. Figure 6 shows a linear conductance $G_{l i n}=\left.\frac{d I}{d(e V)}\right|_{e V \rightarrow 0}$ vs. external magnetic field $B$, calculated within the present approach (top panel) and the standard EOM technique (bottom panel). While both approaches lead to similar behavior of the total conductance $G_{l i n}$, i.e. a maximum at $B=B_{\text {comp }}=0.11$ (indicated by thin vertical line), they give different behavior of spin polarized contributions $G_{\sigma}$ to it. Within the MEOM approach (top panel) the transport is governed by majority spin electrons at small $B$ fields $\left(B<B_{\text {comp }}\right)$, and by minority spin electrons at higher fields $\left(B>B_{\text {comp }}\right)$. Such asymmetry of $G_{\sigma}$ can be explained by the spin state of QD, similarly as the asymmetry of the DOS (see Fig. 1). The peak in $G_{\uparrow}$ (dashed line) steams from the fact that at $B<B_{\text {comp }}$ the QD is occupied by spin up electrons $\left(n_{\uparrow}>n_{\downarrow}\right.$ ) (see Fig. 3), thus the spin up component of the cotunneling current is dominant. At $B>B_{\text {comp }}$ the situation is opposite, namely, $n_{\uparrow}<n_{\downarrow}$, thus spin down cotunneling current is larger. At $B=B_{\text {comp }}$ both spin channels equally contribute to the transport, again, indicating that full Kondo effect is recovered in this case. Note that, the calculated spin polarization vanishes in this case (see Fig. 3). 

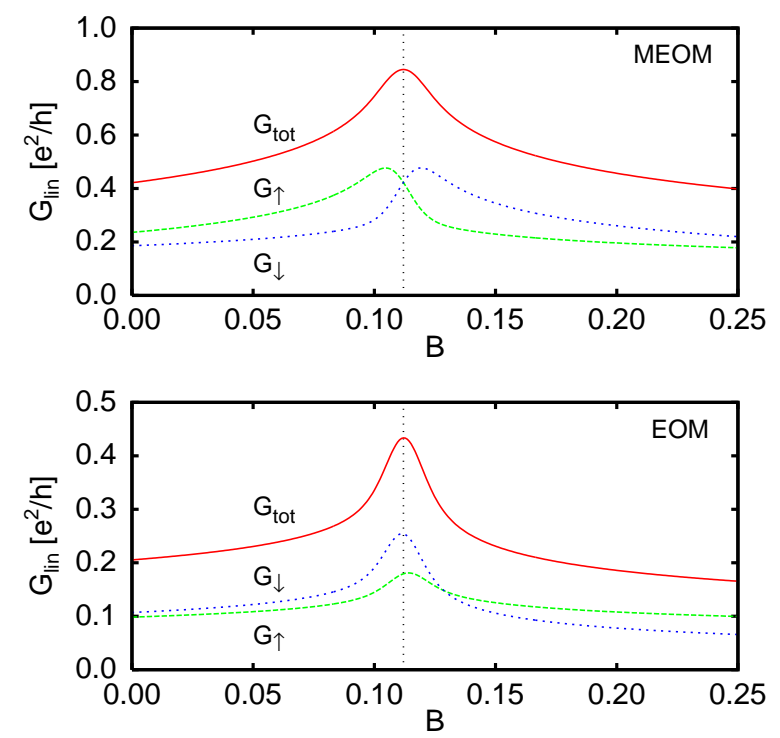

Figure 6. The linear conductance $G_{l i n}$ (solid lines) and spin polarized contributions to it (dashed and dotted lines) vs. magnetic field $B$, calculated within the present approach (top panel) and the standard EOM technique (bottom panel). Note different behavior of $G_{\sigma}$ in both approaches.

Such a behavior of the conductance and the spin polarization remains in good agreement with a real time diagrammatic technique (compare Fig. 6 (a) of Ref. 34]). Within the MEOM approach, the condition $G_{\uparrow}=G_{\downarrow}$ at $B=B_{\text {comp }}$ steams from the fact that the density of states for both spin directions are the same (see Fig. 4) and the couplings to the leads are equal $\left(\Gamma_{\lambda \uparrow}=\Gamma_{\lambda \downarrow}=\Gamma_{\lambda 0}\right)$.

The situation is somewhat worrying in the standard EOM approach (bottom panel), as it gives an opposite behavior of $G_{\sigma}$. At $B<B_{\text {comp }}$, larger contribution comes form minority electrons, and at $B>B_{\text {comp }}$, it comes from majority electrons. Moreover, at $B=B_{\text {comp }}$, the transport still is spin polarized $\left(G_{\uparrow} \neq G_{\downarrow}\right)$. Accidentally, the transport becomes unpolarized at slightly larger field than $B_{\text {comp }}$, while spin polarization vanishes at different filed $B<B_{\text {comp }}$ (compare Fig. 3).

Under non-equilibrium conditions, the discrepancies between standard EOM and MEOM approaches look similar. Figure 7 shows a comparison of the differential conductance $G(\mathrm{eV})=\frac{d I}{d(\mathrm{eV})}$ vs. bias voltage $\mathrm{eV}=\mu_{L}-\mu_{R}$ at zero magnetic field (top panel) and at $B=B_{\text {comp }}=0.11$ (bottom panel), obtained within the present approach (solid lines) and the standard EOM scheme (dashed lines). Both approaches give qualitatively similar behavior of the total conductance, i.e. the same splitting of the zero bias anomaly at zero magnetic field and its lack when the Kondo effect is compensated $(B=0.11)$. However, the present approach gives larger values of the conductance. Moreover, within the present approach (not shown here) both spin channels equally contribute to the transport at any voltage, when the Kondo effect is recovered by the external magnetic field $B=B_{\text {comp }}$. On the other hand, standard EOM 

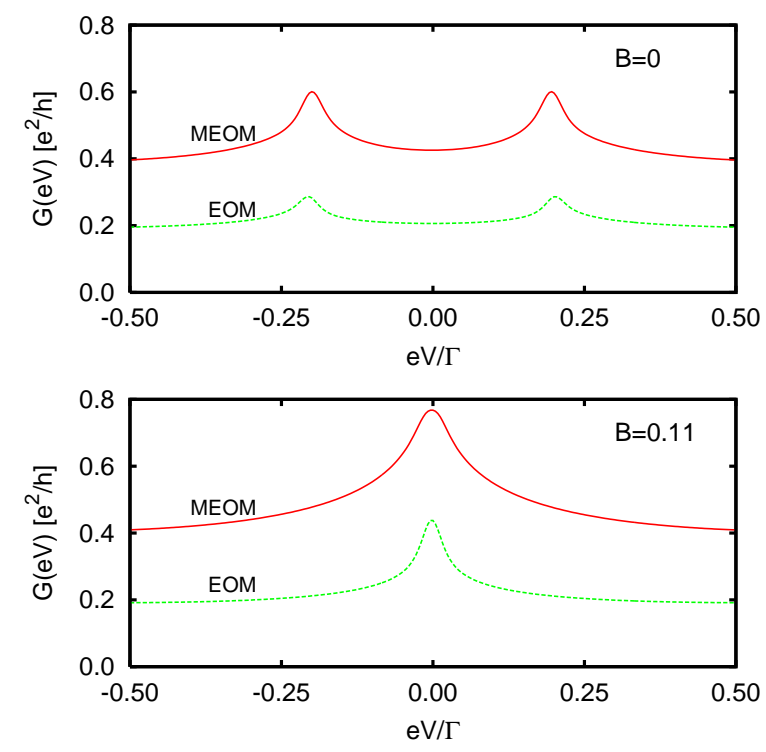

Figure 7. Differential conductance $G(e V)=\frac{d I}{d(e V)}$ vs. bias voltage $e V=\mu_{L}-\mu_{R}$ of a quantum dot coupled to ferromagnetic leads with $\mathrm{p}=0.2$. Top panel represents $G(e V)$ without external magnetic field, while the bottom one is for $B=B_{\text {comp }}=0.11$. Solid lines are obtained with help of the present (MEOM) approach, and dashed lines - within standard EOM technique.

technique leads to a spin polarized $G(\mathrm{eV})$ (see Fig. 2 (d) of Ref. [20]).

Corresponding voltage dependence of the spin polarization is shown in Fig. 8. Again, at zero magnetic field (top panel), both approaches give qualitatively similar behavior of the dot spin polarization for not too large voltages, in agreement with a real time diagrammatic calculations [34. At higher voltages (not shown), in the standard EOM approach the spin polarization weakly increases with $\mathrm{eV}$, while it still decreases in the present approach. On the other hand, when the Kondo effect is compensated by the external $B$ field (bottom panel), standard EOM approach gives non-zero values of the spin polarization, while the modified one gives vanishing of it at $\mathrm{eV}=0$ and very weak increasing with the voltage.

All the above results indicate that the present approach should better describe the compensation of the Kondo effect, as the transport (linear and non-linear conductance) is unpolarized, there is no splitting of the Kondo resonance and spin polarization vanishes in this case, similarly as in other approaches [27, 34]. On the other hand, standard EOM scheme also gives no splitting of the Kondo resonance but at the same time it gives non-zero spin polarization and spin polarized transport properties.

\section{Conclusions}

In conclusion we have proposed simple modifications of the standard equation of motion approach to get a better description of the Kondo effect in a quantum dot coupled to ferromagnetic leads. Special emphasis was put to the compensation of the 

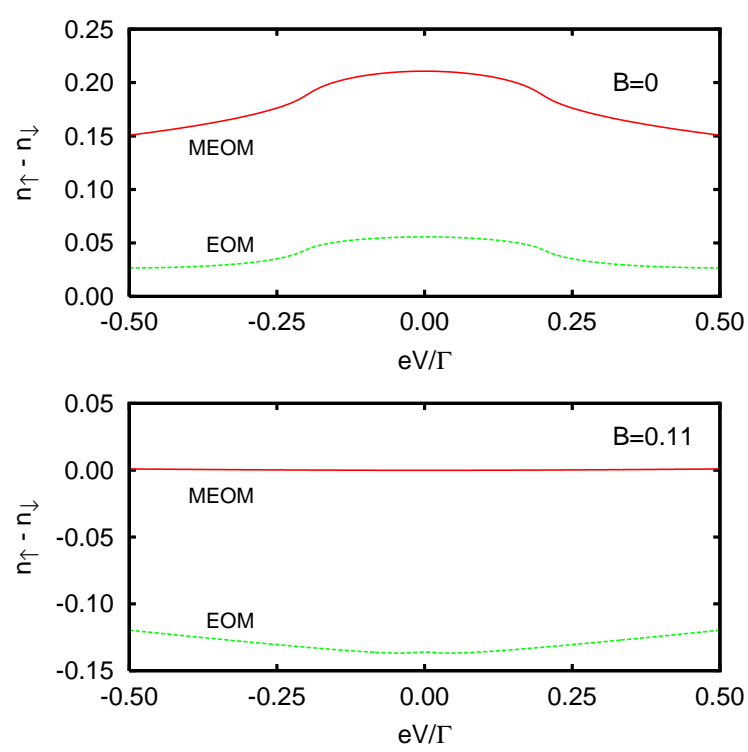

Figure 8. Spin polarization $n_{\uparrow}-n_{\downarrow}$ as a function of applied bias voltage $e V$ in zero magnetic field (top panel) and at $B=B_{\text {comp }}=0.11$ (bottom panel). Solid lines represent the results obtained by MEOM, and dashed lines - by standard EOM approach. Note that within the standard EOM spin polarization does not vanish at $B=B_{\text {comp }}$.

Kondo effect by external magnetic field. In particular, the present approach correctly gives both, no splitting of the Kondo resonance and the vanishing of the dot spin polarization at compensating magnetic field, while the standard equation of motion approach gives non-zero value of the polarization. Moreover, the transport also remains unpolarized within the present approach, while the standard EOM gives not equal spin dependent contributions to the linear and non-linear conductance when the Kondo effect is recovered. In spite of lack of reliable techniques for studying the non-equilibrium transport, the present approach could help us to understand the properties of a quantum dot in the presence of ferromagnatism in the electrodes.

\section{Acknowledgments}

This work has been supported by the Polish Ministry of Education and Science under Grant No. N202 081 31/0372.

\section{References}

[1] G. A. Prinz, Science 282, 1660 (1998).

[2] Semiconductor Spintronics and Quantum Computation, ed. D. Awschalom et al., Springer, New York (2002).

[3] D. Loss, D. P. DiVincenzo, Phys. Rev. A57, 120 (1998).

[4] A. C. Hewson, The Kondo Problem to Heavy Fermions, Cambridge University Press, Cambridge (1993). 
[5] L. I. Glazman, M. E. Raikh, JETP Lett. 47, 452 (1988).

[6] T. K. Ng, P. A. Lee, Phys. Rev. Lett. 61, 1768 (1988).

[7] A. Kawabata, J. Phys. Soc. Japan 60, 3222 (1991).

[8] Y. Meir, N. S. Wingreen, P. A. Lee, Phys. Rev. Lett. 66, 3048 (1991); Phys. Rev. Lett. 70, 2601 (1993).

[9] S. Herschfield, J. H. Davies, J. W. Wilkins, Phys. Rev. Lett. 67, 3720 (1991); Phys. Rev. B46, 7046 (1992).

[10] N. S. Wingreen, Y. Meir, Phys. Rev. B49, 11040 (1994).

[11] M. Krawiec, K. I. Wysokiński, Phys. Rev. B66, 165408 (2002).

[12] D. Goldhaber-Gordon, H. Shtrikman, D. Mahalu, D. Abusch-Magder, U. Meirav, M. A. Kastner, Nature 391, 156 (1998).

[13] S. M. Cronenwett, T. H. Oosterkamp, L. P. Kouwenhoven, Science 281, 540 (1998).

[14] J. Schmid, J. Weis, K. Eberl, K. von Klitzing, Physica B 256-258, 182 (1998); Phys. Rev. Lett. 84, $5824(2000)$.

[15] F. Simmel, R. H. Blick, J. P. Kotthaus, W. Wegscheider, M. Bichler, Phys. Rev. Lett. 83, 804 (1999).

[16] S. Sasaki, S. De Franceschi, J. M. Elzerman, W. G. van der Wiel, M. Eto, S. Tarucha, L. P. Kouwenhoven, Nature 405, 764 (2000).

[17] A. N. Pasupathy, R. C. Bialczak, J. Martinek, J. E. Grose, L. A. K. Donev, P. L. McEuen, D. C. Ralph, Science 306, 85 (2004).

[18] J. Nygard, W. F. Koehl, N. Mason, L. DiCarlo, C. M. Marcus, cond-mat/0410467.

[19] N. Sergueev, Q. F. Sun, H. Guo, B. G. Wang, J. Wang, Phys. Rev. B65, 165303 (2002).

[20] J. Martinek, Y. Utsumi, H. Imamura, J. Barnaś, S. Maekawa, J. König, G. Schön, Phys. Rev. Lett. 91, 127203 (2003).

[21] R. Lu, Z. -R. Liu, cond-mat/0210350.

[22] P. Zhang, Q. K. Xue, Y. P. Wang, X. C. Xie, Phys. Rev. Lett. 89, 286803 (2002).

[23] J. Ma, B. Dong, X. L. Lei, Commun. Theor. Phys. 43, 341 (2005).

[24] B. R. Bułka, S. Lipiński, Phys. Rev. B67, 024404 (2003).

[25] R. López, D. Sánchez, Phys. Rev. Lett. 90, 116602 (2003).

[26] B. Dong, H. L. Cui, S. Y. Liu, X. L. Lei, J. Phys.: Condens. Matt. 15, 8435 (2003).

[27] J. Martinek, M. Sindel, L. Borda, J. Barnaś, J. König, G. Schön, J. von Delft, Phys. Rev. Lett. 91, 247202 (2003).

[28] M. -S. Choi, D. Sanchez, R. Lopez, Phys. Rev. Lett. 92, 056601 (2004).

[29] J. Ma, X. L. Lei, Europhys. Lett. 67, 432 (2004).

[30] J. König, J. Martinek, J. Barnaś, G. Schön, CFN Lectures on Functional Nanostructures, Eds. K. Busch et al., Lecture Notes in Physics 658, Springer, 145 (2005).

[31] J. Martinek, M. Sindel, L. Borda, J. Barnaś, R. Bulla, J. König, G. Schön, S. Maekawa, J. von Delft, Phys. Rev. B72, 121302(R) (2005).

[32] Y. Tanaka, N. Kawakami, J. Phys. Soc. Japan 73, 2795 (2004).

[33] D. Sanchez, R. Lopez, M. -S. Choi, J. Supercond. 18, 251 (2005).

[34] Y. Utsumi, J. Martinek, G. Schön, H. Imamura, S. Maekawa, Phys. Rev. B71, 245116 (2005).

[35] R. Świrkowicz, M. Wilczyński, J. Barnaś, J. Phys.: Condens. Matt. 18, 2291 (2006).

[36] M. Krawiec, K. I. Wysokiński, Phys. Rev. B73, 075307 (2006); Physica B378-380, 933 (2006).

[37] M. Krawiec, J. Phys.: Condens. Matter 18, 6923 (2006).

[38] J. C. Le Guillou, E. Ragoucy, Phys. Rev. B52, 2403 (1995).

[39] M. Krawiec, K. I. Wysokiński, Solid State Commun. 115, 141 (2000).

[40] M. Krawiec, K. I. Wysokiński, Supercond. Sci. Technol. 17, 103 (2004).

[41] H. Haug, A. P. Yauho, Quantum Kinetics in Transport and Optics of Semiconductors, Springer, Berlin (1996).

[42] H. Suhl, D. R. Fredkin, Phys. Rev. 131, 1063 (1963).

[43] Y. Nagaoka, Phys. Rev. 138, A1112 (1965). 
[44] J. A. Appelbaum, D. R. Penn, Phys. Rev. 188, 874 (1969).

[45] C. Lacroix, J. Phys. F11, 2389 (1981).

[46] R. C. Monreal, F. Flores, Phys. Rev. B72, 195105 (2005).

[47] We neglected GFs $\left\langle\left\langle c_{\lambda^{\prime} \mathbf{k}^{\prime}-\sigma}^{+} c_{\lambda \mathbf{k} \sigma} b^{+} f_{-\sigma} \mid f_{\sigma}^{+} b\right\rangle\right\rangle_{\omega}, \quad\left\langle\left\langle c_{\lambda^{\prime} \mathbf{k}^{\prime}-\sigma} c_{\lambda \mathbf{k} \sigma} f_{-\sigma}^{+} b \mid f_{\sigma}^{+} b\right\rangle\right\rangle_{\omega}$, $\left\langle\left\langle c_{\lambda^{\prime} \mathbf{k}^{\prime} \sigma}^{+} c_{\lambda \mathbf{k}-\sigma} f_{-\sigma}^{+} b \mid f_{\sigma}^{+} b\right\rangle\right\rangle_{\omega}$ and set $\left\langle\left\langle c_{\lambda^{\prime} \mathbf{k}^{\prime}-\sigma}^{+} c_{\lambda \mathbf{k}-\sigma} b^{+} f_{\sigma} \mid f_{\sigma}^{+} b\right\rangle\right\rangle_{\omega}=\delta_{\lambda \lambda^{\prime}} \delta_{\mathbf{k} \mathbf{k}^{\prime}} f\left(\epsilon_{\lambda \mathbf{k}-\sigma}\right) G_{\sigma}^{r}(\omega)$, in the second iteration of equation of motion for $G_{\sigma}^{r}(\omega)$

[48] H. Keiter, J. C. Kimball, J. Appl. Phys. 42, 1460 (1971); N. Grewe, H. Keiter, Phys. Rev. B24, 4420 (1981); Y. Kuramoto, Z. Phys. B53, 37 (1983).

[49] T. A. Costi, J. Kroha, P. Wölfle, Phys. Rev. B53, 1850 (1996).

[50] M. H. Hettler, J. Kroha, S. Hershfield, Phys. Rev. B58, 5649 (1998).

[51] M. Pustilnik, Y. Avishai, K. Kikoin, Phys. Rev. Lett. 84, 1756 (2000); M. Eto, Yu. V. Nazarov, Phys. Rev. B64, 085322 (2001). 